\title{
A Study on Composition Goal in Automated Web Services Composition Approaches
}

\author{
V.Portchelvi \\ Research Scholar \\ Dept. of Banking Technology \\ Pondicherry University \\ Pondicherry, South India
}

\author{
V. Prasanna Venkatesan \\ Associate Professor \\ Dept. of Banking Technology \\ Pondicherry University \\ Pondicherry, South India
}

\author{
G. Shanmugasundaram \\ Research Scholar \\ Dept. of Banking Technology \\ Pondicherry University \\ Pondicherry, South India
}

\begin{abstract}
Automated Web Services Composition (AWSC) has received more attention in the research community. In the literature, automated composition of web services problem has been identified to find approaches that realize a particular request by detecting, composing and executing web services automatically. Goal-driven Architecture provides a promising solution to this problem. It allows a composition system to formulate a request in terms of a goal and solves it by automatically discovering, composing and executing appropriate web services. The main element of this architecture is the concept of goal because a goal carries all the necessary information for automated discovery, composition and execution of web services. Our aim is to study the Automated Web services composition approaches based on this architecture and to analyze them based on the concept of goal.
\end{abstract}

\section{General Terms}

Software Architecture, Service-Oriented Architecture, Web Services.

\section{Keywords}

Web Services composition, AI Planning, Goal, Goal-Driven Architecture, Automated Composition Approaches.

\section{INTRODUCTION}

In order to realize the automated Business-to-business interactions, web services composition is required wherein many services belonging to different enterprises are composed together and executed to realize complex business functionality. Web services composition must enable us to compose services automatically on- the- fly and on demand in order to realize business processes that can be created on demand. The benefit is virtual enterprises are created dynamically which shorten delivery times, increase product quality, deliver personalized services, decrease transaction costs, and accommodate short-term cooperating relationships.

In the literature, most of the service composition approaches concentrate much on the automated generation of on-demand service composition process specification. In order to achieve automated on-demand service composition, researchers related this to the automated problem solving techniques available in AI. There are lot of frameworks that are developed by different branches of Artificial Intelligence (AI) research in order to describe problems and resources along with technologies for automated problem resolution and resource usage. These frameworks support both client side as well as service side. On the service side, it helps in defining the computational resources such that they can be allowed in automated usage. On the client side, it helps in describing the problem to be solved and the resolution process. The client side allows the client to specify his objectives from his perspective without considering the technical details. The client side description must provide necessary information required to the intelligent mechanism for automated problem resolution. The information provided by the client side aids in automated resource detection, combination and usage. An architecture that supports this type of client side descriptions is called goal-driven architectures. This architecture allows, the user to specify only his wish or the problem to be solved and the intelligent mechanisms solves the problem automatically without any human intervention. The goaldriven architecture [1] mainly focuses on the client side rather than the service side. The reason is, only the client side provides necessary required information to the intelligent mechanisms for automated problem solving. The main element of this architecture is the concept of goal because a goal carries all the necessary information for automated discovery, composition and execution of web services.

\section{OBJECTIVE}

The two major approaches that pave way for achieving AWSC are AI-Planning based approach and Goal-Driven approach. These approaches rely much on Goal-Driven Architecture for AWSC and the goal is the major element in this architecture which carries all the necessary information for solving AWSC problem. Since the goal is the only element in these approaches which provides the necessary required information to the intelligent mechanisms for achieving AWSC, it motivated us to study and explore this element. This study will enable us to understand the client side of these two approaches and will also make us understand the level to which a goal has been specified in these approaches.

\section{GOAL-DRIVEN ARCHITECTURE}

Goal-driven architectures provide the basis for Automated AWSC approaches. The main element of this architecture is the concept of goals. As the central element of such architectures, a goal has been generally defined in [1] as a formal, machineprocess able knowledge level specification of a client objective. A goal must abstract the technical details and allow the client to specify his request in a natural way. A goal must allow a client to specify all possible types of objectives he has. Moreover a goal must provide all the necessary information required for the automated goal resolution. Therefore it is understood that in automated service composition process a composition goal takes into account the user's constraints and requirements which he/she specifies in her or his request. Then based on this request, composition takes place by combining the component web services appropriately. To make this possible in an automatic way, the composition goals and 
services must be represented and made in machine understandable format. Since automated composition process starts from the description of composition goal, it is necessary to focus on the concept of composition goal. Most realization approaches in the literature assume that a well formulated goal is available and concentrates on the other part, automated resolution of composition goal. Section 4 provides an insight into the description of composition goal in existing automated composition approaches.

\section{GOAL DESCRIPTION}

\subsection{AI-Planning based Approaches}

A great deal of work has been done in the area of automated service composition. AI-planning techniques are predominantly utilized to achieve automated service composition. For automated problem solving, planning depends upon the Goal-Driven Architecture which provides a promising solution to this problem. The main element of this architecture is the concept of goals. Goals in this architecture provide necessary information to resolve them automatically. AI Planning technique uses this automated goal resolution technique to generate composition specification automatically based on user request in web services composition domain. AI-Planning based approaches take the automated service composition problem as a planning problem and generates a composition plan. It uses Goal-Oriented Action Planning (GOAP) to construct a composition plan which takes an abstract goal and resolves it automatically. In these approaches a composition goal is specified in terms of Input, Output, Pre-conditions and Effects (IOPE). A detailed study about how Goals are viewed in AI-Planning based Service Composition Approaches is done and shown in Table 1.This goal specification not only specifies the final state that should be reached but also describes constraints on the overall properties of a composite service. In AI- planning based approaches, on the client side, the user specifies his objectives to be achieved and the intelligent planning algorithm takes the objective of the client as goal and produces a plan for the goal resolution process. A plan contains a sequence of actions that will lead to the goal. These approaches view the goal as a planning goal rather than a composition goal. All these approaches do not have a generic description of a goal for composition. Hence a composition goal description for these approaches could be generalized and viewed as shown in Table 2.

\subsection{Goal-driven Approach}

A goal in AI-Planning based AWSC approaches is not suitable for non-technical end users to specify requirements because user-friendly methods for the specification of initial states, goal states and service capabilities (required to generate automated composition process model) are missing. These works have mostly concentrated on only one or two aspects of the composition problem. Most of the literature survey in this area focuses on the planning aspect of web services composition. Therefore it is clear that the AIPlanning based approaches have not given attention towards non-technical end-users for formulating their request in a nontechnical way. Due to the less attention given to the specification of non-technical end-users requirements in AIPlanning based ASWC approaches, Goal-driven approaches based on the concept of goal for specifying user requirements started emerging. In this approach, an end-user shall give his/her request in terms of a knowledge level goal and the system solves this goal by automatically discovering, composing and executing appropriate web services. These approaches exhibit a Goal-driven behavior by which they allow a composition system to accept high-level (Knowledge level) goals. They enable the end-users to have a feel of formulating their request at problem level and not at a technical level. To achieve this composition goal in this approach has to be modeled as shown in Table 3.

\section{GOAL ANALYSIS}

\subsection{AI-Planning based Approaches}

Literature on goal-based services composition approach discusses about the drawbacks of AI Planning based service composition approach. The most promising AI planning approaches discussed in [2] and [3] has been taken for discussion and in [2] Goals are ground goals rather than knowledge level goals, the Goal model is state-oriented goal model and goal is resolved by Goal-Oriented Action Planning (GOAP). In [3], the goal is given as a high-level task. The planner decomposes this high-level task into subtasks using some decomposition methods. The user provides explicitly a partially ordered set of tasks instead of the goal formula. Hence this planning approach could not be referred to as a fully automated approach because the user composes the services manually in terms of tasks. The goal model adopted in these planners is task-oriented goal model.

\subsection{Goal-driven Approach}

In [4][5][6]Goal-driven service composition is discussed. Some attempt has been made in the literature to specify the user criteria in terms of goals [6] by making the non technology literates to express their requirements in an easier way (natural language). A Goal based service ontology and domain ontology are used to transform the goal expressed in natural language into formal goal. In [4], from the user request, goal is extracted and decomposed and a goal tree has been built. The size of the tree is determined by the specific requirements and preferences provided by the user in his/her request. Then the goal described is processed by a semantic reasoner which decomposes the main goal into more primitive goals. A composition plan is generated based on the determined sub goals and their relationships and does not contain any concrete services. Petri net is used to represent this plan and the plan is filled with concrete services. This composition is analyzed, verified and it is ready for implementation. In [5], the requirements are captured in a goal model. The goal model captures the functional requirements which state the functionality of the system to be, non-functional requirements that state how to keep the system on track in case of anomalies and requirements that enable the system to be self-adaptive. Modified KAOS goal model is used to offer explicit support for the definition of adaptive goals (i.e. when to apply adaptation and enforce the actions regarding the adaptation).

\section{DISCUSSION}

In this paper a detailed survey has been done on Automated Web services composition approaches based on the concept of goals. It has been noted that for automated discovery and composition of web services, AI Planning technique based approaches are used. These approaches solve composition problem by mapping it to a planning problem. In these approaches web services are modeled as actions, business process as a plan connecting the web services and desired service as goal. These approaches assume that a business process or application is associated with some explicit goal definition. An AI planner takes this explicit goal and determines the combination of actions needed to reach the 
goal. But explicit goals are usually not available from an industrial perspective [8]. It is strongly argued that in BPI and EAI domains where web services composition is required, explicit goals are not given. It has been identified that the major drawback of AI planning based approaches is the explicit goal definition and it is questioned that how the goals for automatic composition can be derived [9]. To solve these drawbacks, Model Driven Architecture for Web services composition is adopted. In this approach, abstract workflows are generated from business models and code is generated automatically.

Though an explicit goal definition is required for automated composition, in most of the AI planning based approaches a goal denotes a situation that should be reached. In real business scenarios, automated discovery and composition of web services is relative to user's criteria and a technique is required to specify this user's criteria. Existing approaches use IO and IOPE parameters [12] to specify composition requirements and uses formal semantics for automated discovery and composition of web services which leads to difficulty in developing automatic discovery and composition algorithms. As a result of this, a goal specification reduces to the types of Inputs and Outputs parameters. But the real requirement is to allow a requestor searching for a composite web service to describe his preferences and constraints on the overall properties of a composite web service and not only a final state that should be reached. This requires an expressive goal that allows the specification of relationships between inputs and outputs and temporal constraints on the output. Therefore techniques for expressing goals and expressive formalism for modeling expressive goals are required [7]. An attempt has been made in [7] to develop an expressive formalism for modeling an above mentioned goal and leaves the development of automatic composition of web services an interesting and open research problem.

These approaches view the goal as a planning goal rather than a composition goal. It is clear that the AI-Planning based approaches have not given attention towards non-technical end-users for formulating their request in a non-technical way. In Goal-driven approach some attempt has been made to specify the user criteria in terms of goals [6] by making the non technology literates to express their requirements in an easier way (natural language). A Goal based service ontology and domain ontology are used to transform the goal expressed in natural language into formal goal. But this approach is suitable only for environments where the domain is clear and well known. [4] allows the user to request the composite service in terms of high level goal and the system extracts the semantic specification of the goal with the help of domain ontologies and interpret the goal and its attributes based on them. In [5], adaptive goals are used to provide reliable service composition. Adaptive goals are to dynamically trigger adaptation actions, cause the system to move to a new goal model and propagate the corresponding changes to the underlying implementation.

\section{CONCLUSION}

A detailed study about how Goals are viewed in Automated Web Services Composition Approaches is done. In AIPlanning based approaches, a goal has been viewed at micro level i.e. it has been used to generate automated composition plan. From this study we are able to understand that these approaches view the goal as a planning goal rather than a composition goal. In Goal-driven approaches, a goal has been viewed at macro level i.e. it allows the user to express their requirements in non-technical and user-friendly ways and it is transformed into a formal goal with the help of a goal ontology or domain ontology. Therefore we are able to conclude that most of the AI planning based automated service composition approaches in the literature concentrates much on the automatic generation of plans and they assume that a well formulated goal is available. The concept of goal has been overlooked in these approaches. And the main drawback of Goal-driven approaches is they are suitable only for environments where the domain is clear and well known. Achieving AWSC is a challenging task [14] [13] [15] and AWSC is a process that starts from a composition goal and ends into an appropriate combination of component Web Services. But the approaches for AWSC do not have a generic description of a composition goal. According to this study the formulation of a Composition Goal is very important to achieve AWSC and it is found that it has been overlooked in AWSC approaches that need some research attention. We believe that the concept of goal can be exploited fully in all the stages of composition that could provide promising solution to the requirement of having a high-level service composition framework that addresses the composition process from start to finish. This aims for an agile service composition approach which provides flexibility on both the client side as well as the service side.

\section{REFERENCES}

[1] Michael Stollberg, Martin Hepp , July 10, 2006, WP3 Service Ontologies and Service Description D3.10 Goal Description Ontology.

[2] P. Traverso \& M. Pistore ,2004, Automated Composition of Semantic Web Services into Executable Processes. In: Proc. of ISWC-04, LNCS 3298. Springer, pp. 380-394.

[3] Wu, D., Sirin, E., Hendler, J., Nau, D., and Parsia, B. 2003. Automatic Web Services Composition Using SHOP2. Twelfth World Wide Web Conference.

[4] Dmytro Zhovtobryukh, 2007, A Petri Net-based Approach for Automated Goal-Driven Web Service Composition, $\quad$ SIMULATION, $83 ; \quad 33$

[5] Liliana Pasquale, 2007, From Goals to Reliable Service Compositions, This research has been partially funded by the European Commission, Programmes: IDEAS-ERC, Project 227977 SMScom, and FP7/2007-2013, Projects 215483 S-Cube (Network of Excellence) and 216556 SLA@SOI.

[6] Luiz Olavo Bonino da Silva Santos, Giancarlo Guizzardi, Renata Silva Souza Guizzardi,Eduardo Gonc alves da Silva, Lu'ss Ferreira Pires and Marten van Sinderen, GSO: Designing a Well-Founded Service Ontology to Support Dynamic Service Discovery and Composition.

[7] Sudhir Agarwal, 2007, A Goal Specification Language for Automated Discovery and Composition of Web Services, in Proc. of International Conference on Web Intelligence, 2007 IEEE/WIC/ACM

[8] Biplav Srivastava and Jana Koehler, 2003, Web service composition - current solutions and open problems, in Proc. Of International conference on Automated Planning and 2003. 
[9] Biplav Srivastava and Jana Koehler, 2004, Planning with workflows - An emerging paradigm for web services composition, in Proc. Of International conference on Automated Planning and Scheduling, 2003.

[10] Zaiwen Feng, Keqing He, Yutao Ma, Rong Peng, Ping Gong, 2008, A Requirements-Driven and AspectOriented Approach for Evolution of Web Services Composition.

[11] Maja Vukovi'c, IBM T.J.Watson Research, Evangelos Kotsovinos, Peter Robinson, Application development powered by rapid, on-demand service composition.
[12] Peter Bartalos,2010, Effective automatic dynamic semantic web service composition, ACM

[13] Peer, J., 2005 Web service composition as AI planning a survey. Technical report, Univ. of St. Gallen, Switzerland.

[14] Juan Carlos Zũniga, Jos'e J. P'erez-Alc'azar, Luciano Digiampietri, 2010, Implementation Issues for Automatic Composition of Web Services, 2010 Workshops on Database and Expert Systems Applications.

[15] M. P. Papazoglou et al., 2007, Service-Oriented Computing: State of the Art and Research Challenges, Computer, vol. 40 , no. 11 , pp. 38-45.

Table 1. Goals in AI based Planning Service Composition Approaches

\begin{tabular}{|c|c|c|c|}
\hline S.No. & Planner & Goal & Drawback \\
\hline 1 & $\begin{array}{l}\text { Simple Hierarchical Order Planning } \\
\text { (SHOP) [ 3] }\end{array}$ & $\begin{array}{l}\text { Accepts goal as task lists } \\
\text { - Goal tasks } \\
\text { - primitive tasks } \\
\text { - compound tasks }\end{array}$ & \multirow{2}{*}{$\begin{array}{l}\text { - How to express non- } \\
\text { determinism and iterations in } \\
\text { compound tasks is still an } \\
\text { unexplored issue. } \\
\text { Carries out a given task } \\
\text { (complex or primitive) task } \\
\text { but do not plan to achieve a } \\
\text { defined goal. }\end{array}$} \\
\hline 2 & SHOP2 Planner [3 ] & $\begin{array}{l}\text { Supports Extended goals. } \\
\text { Composite tasks specification } \\
\text { describes the changes required } \\
\text { by the user and thus the } \\
\text { extended goals are achieved. }\end{array}$ & \\
\hline 3 & NDSHOP2 [ 3] & $\begin{array}{l}\text { User request contains the } \\
\text { desired goal, application } \\
\text { domain and some constraints. } \\
\text { Each domain has its own } \\
\text { specific goals. Goals in the } \\
\text { domain are specified using } \\
\text { ontological language used in } \\
\text { the domain. It has to be } \\
\text { translated to the planner's } \\
\text { language. }\end{array}$ & $\begin{array}{l}\text { The task that has to be performed by the } \\
\text { planner must be stated explicitly. This is } \\
\text { not suitable for dynamic environments. }\end{array}$ \\
\hline 4 & $\begin{array}{l}\text { Planning as Model Checking } \\
\text { PMB[2] }\end{array}$ & $\begin{array}{l}\text { Composition Goal provides the } \\
\text { requirements for the composite } \\
\text { service that has to be generated } \\
\text { automatically. } \\
\text { Supports Extended goals with } \\
\text { both safety condition and } \\
\text { liveness condition. } \\
\text { Supports the specification of } \\
\text { requirements of different } \\
\text { strengths and preference } \\
\text { conditions. } \\
\text { Input is composition goal and } \\
\text { planning domain. }\end{array}$ & $\begin{array}{l}\text { Reasoning techniques for discovery and } \\
\text { selection of OWL-S service profiles are } \\
\text { not available. } \\
\text { Automata-like representations were } \\
\text { focusing on the representation of goals } \\
\text { and not actions. } \\
\text { Composition plan is generated } \\
\text { completely at the beginning before the } \\
\text { execution. As goals get incrementally } \\
\text { refined, composition plan generation } \\
\text { must be a continual process. }\end{array}$ \\
\hline 5 & TLPLAN[11] & Temporally extended Goals & $\begin{array}{l}\text { No special mechanisms for quantified } \\
\text { goals. For Goals with complex time } \\
\text { constraints the planner does not utilize } \\
\text { any special temporal reasoning. }\end{array}$ \\
\hline
\end{tabular}


Table 2. Composition Goal description for AI-Planning based AWSC

\begin{tabular}{|c|c|c|}
\hline Need & Purpose & Composition Goal \\
\hline Specifying objectives & $\begin{array}{l}\text { to allow expressing more complex objectives, a } \\
\text { goal description model should allow specifying } \\
\text { additional constraints for goal resolution in terms } \\
\text { of constraint types C-I as well as desired } \\
\text { workflows for service aggregation in form of C-II } \\
\text { constraints. }\end{array}$ & $\begin{array}{l}\text { Desired states of the world (G-I) } \\
\text { Functions to be performed (G-II) }\end{array}$ \\
\hline Specifying constraints & & $\begin{array}{l}\text { Constraints that are implicitly hidden with the } \\
\text { domain knowledge in AI Planning (C-I) } \\
\text { Constraints that are specified in conditional AI } \\
\text { planning language (CII). This type refers to } \\
\text { constraints on the goal resolution process. }\end{array}$ \\
\hline Goal Resolution Techniques & $\begin{array}{l}\text { Resolving the goal automatically without human } \\
\text { intervention. }\end{array}$ & A priori plan determination \\
\hline
\end{tabular}

Table 3. Composition Goal description in Goal-Driven AWSC Approach

\begin{tabular}{|c|c|c|}
\hline Need & Purpose & Composition Goal \\
\hline Specifying objectives & \multirow{2}{*}{$\begin{array}{l}\text { to allow expressing more complex objectives, a } \\
\text { goal description model should allow specifying } \\
\text { additional constraints for goal resolution in terms } \\
\text { of constraint types C-I as well as desired } \\
\text { workflows for service aggregation in form of C-II } \\
\text { constraints. }\end{array}$} & G-I,G-II \\
\hline Specifying constraints & & C-I,C-II \\
\hline \multicolumn{3}{|l|}{$\begin{array}{l}\text { Aspects required for } \\
\text { automated goal resolution }\end{array}$} \\
\hline $\begin{array}{l}\text { 1. Automated Detection of } \\
\text { directly usable web services } \\
\text { for solving a goal }\end{array}$ & $\begin{array}{l}\text { Keyword-based matching } \\
\text { Semantic matchmaking } \\
\text { Selection based on QoS aspects }\end{array}$ & $\begin{array}{l}\text { Keywords } \\
\text { Formal specification of the requested } \\
\text { functionality } \\
\text { Constraints on QoS aspects }\end{array}$ \\
\hline \multirow[t]{2}{*}{ 2. Automated composition } & $\begin{array}{l}\text { Functional level composition composes the atomic } \\
\text { services and executes in a single request-response } \\
\text { step. }\end{array}$ & IO \\
\hline & $\begin{array}{l}\text { Process level composition is a composition that } \\
\text { takes into account that executing a web service } \\
\text { requires interactions that may involve different } \\
\text { sequential, conditional and iterative steps. }\end{array}$ & IOPE \\
\hline $\begin{array}{l}\text { 3.Communication } \\
\text { conformance }\end{array}$ & $\begin{array}{l}\text { Requirement of a communication structure by the } \\
\text { client for consuming a Web service and interacting } \\
\text { with it. }\end{array}$ & Client interface \\
\hline 4.Execution & $\begin{array}{l}\text { Information required for invoking and executing } \\
\text { the web services }\end{array}$ & Concrete inputs for executing Web services \\
\hline
\end{tabular}

